\title{
Infiltrado pulmonar por trastuzumab: un cuadro de difícil diagnóstico etiológico
}

\author{
L. Heras López ${ }^{1}$, I. Español Morales ${ }^{1}$, F. Losa Gaspà ${ }^{1}$ J. Janariz Roldán',
} E. Bachs Carré2 L. Camacho Cuartero

\section{Resumen}

- Introducción: la aparición de un infiltrado pulmonar difuso en el curso de un tratamiento antineoplásico puede obedecer a múltiples causas, desde las propiamente debidas a la enfermedad neoplásica a las producidas por el tratamiento citostático. La toxicidad pulmonar inducida por trastuzumab es un efecto secundario apenas descrito

- Material y método: presentamos un caso de infiltrado pulmonar bilateral en una paciente con cáncer de mama tratada con docetaxel-trastuzumab. Tras descartar otras causas, se atribuyó el cuadro clínico al trastuzumab. La paciente evolucionó favorablemente tras la suspensión del tratamiento y la administración de glucocorticoides.

- Conclusiones: el diagnóstico de infiltrado pulmonar por trastuzumab es un diagnóstico de exclusión y no existe un tratamiento definido más allá de la suspensión del fármaco.

\section{Palabras clave:}

Infiltrado pulmonar. Trastuzumab. Docetaxel.

Oncología, 2005; 28 (4):207-211

\footnotetext{
${ }^{1}$ Servicio de Oncología

${ }^{2}$ Servicio de Anatomía Patológica

Hospital General de l'Hospitalet

Hospitalet de Llobregat. Barcelona
} 


\section{Summary}

- Introduction: The appearance of a bilateral lung infiltrate during an antineoplastic treatment may be due to an important number of causes: causes related to the disease or related to the treatment.

Lung toxicity is not usual during trastuzumab treatment.

- Material and methods: We describe a case of lung infiltrate in a patient with advanced breast cancer during treatment with docetaxel and trastuzumab. After excluding other causes, we attributed the case to trastuzumab. The patient had a favourable evolution with treatment discontinuation and glucocorticosteroids.

- Conclusions: The diagnosis of a lung infiltrate caused by trastuzumab is an exclusion diagnosis and we don't know about other treatment than stopping trastuzumab administration.

Key words: Lung infiltrate. Trastuzumab. Docetaxel.

\section{Introducción}

La combinación de docetaxel y trastuzumab en el tratamiento de cáncer de mama es activa y bien tole$\operatorname{rada}^{1}$. La toxicidad de este esquema es fácilmente manejable y se trata fundamentalmente de toxicidad hematológica. La toxicidad pulmonar, en cambio, es poco frecuente y secundaria en la mayoría de casos a docetaxel en forma de derrame pleural.

La aparición de un infiltrado pulmonar bilateral en un paciente con cáncer plantea múltiples diagnósticos diferenciales. En un paciente que recibe docetaxel-trastuzumab las principales etiologías a tener en cuenta son la comorbilidad pulmonar basal, las infecciones pulmonares por gérmenes oportunistas debidas a la inmunosupresión, la insuficiencia cardíaca secundaria a trastuzumab o la toxicidad directa por trastuzumab.

El caso que presentamos desarrolló, además de derrame pleural por docetaxel, un infiltrado pulmonar atribuible a trastuzumab que remitió tras la suspensión de éste.

A propósito de este caso realizamos una revisión de la toxicidad pulmonar de esta combinación en la literatura.

\section{Caso clínico}

Mujer de 67 años de edad con antecedentes de hipertensión arterial, insuficiencia cardíaca grado II de la New York Heart Association (NYHA) y fibrila- ción auricular crónica. En Abril de 2004 fue diagnosticada de carcinoma ductal infiltrante de mama izquierda estadío clínico IIIA (T3N1M0). Se realizó mastectomía y linfadenectomía axilar izquierda. El informe anatomopatológico fue de carcinoma ductal infiltrante de $75 \mathrm{~mm}$ de diámetro máximo, 4 ganglios afectos de 9 , receptores hormonales negativos y sobreexpresión de HER2.

En el control postoperatorio se detectaron adenopatías en fosa supraclavicular izquierda y la tomografía axial computerizada (TAC) torácica evidenció además adenopatías subpectorales y axilares homolaterales. Dada la contraindicación para administrar antraciclinas por la cardiopatía subyacente (fracción de eyección de ventrículo izquierdo [FEVI] del $46 \%$ ) y al tratarse de un tumor con sobreexpresión de HER2, se planteó quimioterapia con docetaxel y trastuzumab semanales a las dosis de docetaxel 35 $\mathrm{mg} / \mathrm{m}^{2}$ y trastuzumab $2 \mathrm{mg} / \mathrm{kg}$ precedido de una dosis de carga de $4 \mathrm{mg} / \mathrm{kg}$. Durante las primeras semanas de tratamiento la paciente presentó una toxicidad tolerable, con astenia y onicolisis como trastornos principales. Tras la $10^{\mathrm{a}}$ semana de tratamiento la paciente ingresó por disnea de mínimos esfuerzos de instauración progresiva, tos seca y dolor centrotorácico. En la exploración física destacaba taquipnea con auscultación respiratoria normal, sin signos de fallo cardíaco ni fiebre. Presentaba asimismo onicolisis severa y epífora. La radiografía de tórax mostró un infiltrado bronquioloalveolar difuso de predominio derecho y derrame pleural izquierdo. En la analítica existía una hipoalbuminemia leve, siendo el 


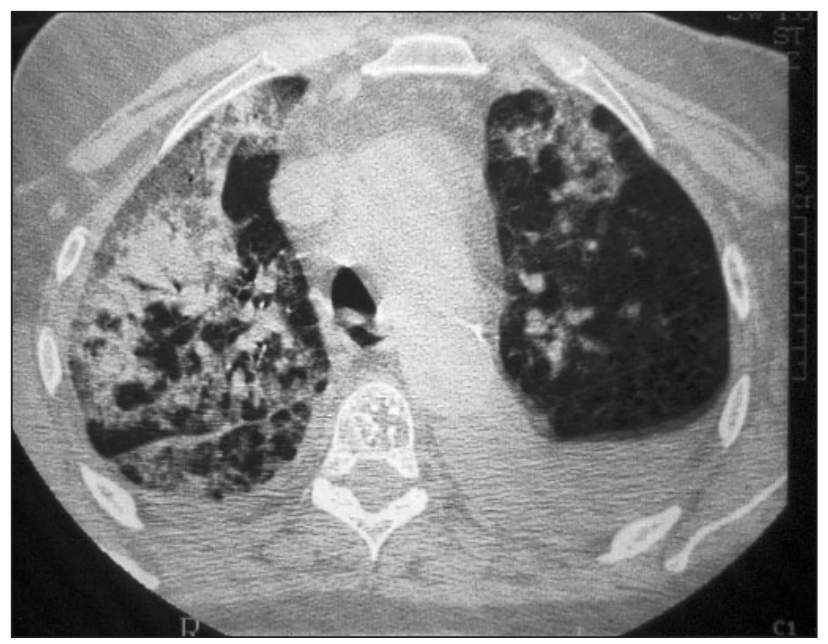

Figura 1. TAC torácica que muestra en parénquima pulmonar derecho una extensa afectación de distribución central, con broncograma en su interior y numerosas áreas de alveolitis. Afectación de menor intensidad en parénquima pulmonar izquierdo y derrame pleural bilateral de predominio izquierdo.

resto anodino. La gasometría mostró hipoxemia e hipocapnia $\left(\mathrm{pO}_{2} 61, \mathrm{pCO}_{2} 31\right)$ y el electrocardiograma la fibrilación auricular ya conocida a 110 por minuto. Ante la sospecha de que pudiera existir una descompensación cardíaca secundaria al tratamiento con trastuzumab se repitió un ecocardiograma que no mostró disminución de la FEVI respecto al estudio basal. La TAC de tórax evidenció el infiltrado brocoalveolar de predominio derecho, además de derrame pleural bilateral y moderado derrame pericárdico (Fig. 1). Por otra parte, se constató una remisión completa de las adenopatías axilares, subpectorales y supraclaviculares. Se completó el estudio con broncoscopia y toracocentesis para descartar progresión de la enfermedad o infección oportunista. El análisis microbiológico y citológico de las muestras bronquiales y del líquido pleural fue negativo. En el lavado brocoalveolar se observaron células bronqioloalveolares hiperreactivas sugestivas de daño celular (Fig. 2). Dada la negatividad de todas las exploraciones realizadas se orientó el caso como posible toxicidad pulmonar por trastuzumab y se inició tratamiento con glucocorticoides a dosis de 1 $\mathrm{mg} / \mathrm{kg}$ de peso con rápida mejoría de la clínica respiratoria y lenta resolución del infiltrado radiológico. La paciente precisó suspensión del tratamiento citostático y recibió radioterapia sobre pared torácica, región axilar y supraclavicular para consolidación de la respuesta.

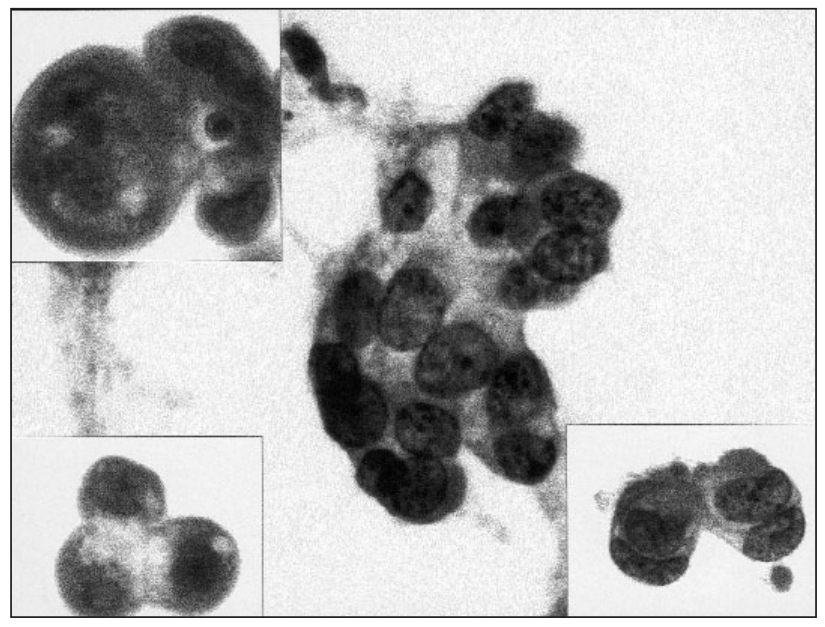

Figura 2: Placa de células bronquioloalveolares regenerativas observadas en caso de daño alveolar. Recuadro: detalle de las mismas con cambios hiperreactivos secundarios al tratamiento (multinucleación y vacuolización citoplásmica) (aumento original:600; tinción: Papanicolau).

\section{Discusión}

La combinación de docetaxel y trastuzumab ha sido recientemente aprobada por la FDA y la EMEA como tratamiento de primera línea del cáncer de mama metastático con sobreexpresión de HER2 ${ }^{1}$. Esta combinación ha demostrado ser activa en varios estudios fase II obteniendo tasas de respuesta de 50$67 \%$ y medianas de supervivencia de 17.8-34 meses $^{2-5}$. La superioridad de esta combinación en comparación con docetaxel solo ha sido demostrada en el ensayo aleatorio fase II M770016 ${ }^{6}$. La adición de trastuzumab a docetaxel mejoró las tasas de respuesta (del 34\% al 61\%, p =0,0002), la mediana de tiempo a la progresión (de 5,7 a 10,6 meses, $\mathrm{p}=$ 0,0001 ) y la mediana de supervivencia (de 22,1 a 30,5 meses, $p=0,0062$ ). La toxicidad por añadir trastuzumab fue escasa y la mayoría de efectos secundarios fueron atribuibles a docetaxel. En los estudios de combinación se han utilizado distintas pautas de administración de docetaxel y diferentes dosis de premedicación con glucocorticoides, lo cual determina posiblemente distintos perfiles de toxicidad. La pauta de administración de docetaxel más utilizada es la semanal ${ }^{2-4}$. En esta forma de administración se ha descrito una mayor incidencia de onicolisis y epífora ${ }^{7}$. En el estudio de Esteva ${ }^{2}$ se describe un $93 \%$ de epífora grado 1-2. La menor dosis de corticoides en la premedicación utilizada en este 
mismo estudio podría explicar también la mayor incidencia de derrame pleural (29\%); de cualquier forma, la dosis óptima de corticoides en pacientes que reciben un régimen semanal de docetaxel no está bien definida. Nuestra paciente presentó onicolisis y epífora severas, congruentes con la pauta semanal de docetaxel, y derrame pleural, posiblemente por la falta de cumplimiento en la premedicación corticoidea.

En cuanto a la toxicidad pulmonar, se reportan pocos casos en estos ensayos. En el estudio de Tedesco $^{4}, 26$ pacientes con cáncer de mama metastático HER-2-positivo recibieron Docetaxel semanal y Trastuzumab. 10 pacientes presentaron disnea (1 caso grado 4) de origen multifactorial (anemia, aumento de peso, derrame pleural) pero en ninguna de ellas se describe un infiltrado pulmonar. En el estudio fase II publicado por Raff ${ }^{3}, 52$ pacientes con cáncer de mama metastático se randomizaron a recibir docetaxel semanal o docetaxel-trastuzumab semanal si sobreexpresaban HER2. 26 pacientes (50\%) presentaron toxicidad respiratoria (6 casos grado 3-4). 2 pacientes requirieron suspensión del tratamiento, 1 por disnea grado 3 y 1 por infiltrado pulmonar grado 1. El caso de infiltrado pulmonar apareció en el grupo que recibió docetaxel solo.

En la literatura sólo hemos encontrado un caso de infiltrado pulmonar en el que la asociación a trastuzumab esté bien fundamentada y en el que además se describe la alteración anatomopatológica subyacente. Se trata del caso que publicó Radzikowska ${ }^{8}$ en una paciente que recibió trastuzumab como tratamiento adyuvante de cáncer de mama. Tras seis semanas de tratamiento con trastuzumab, la paciente presentó un cuadro de disnea, tos seca, fiebre e infiltrado pulmonar bilateral La biopsia pulmonar mostró un patrón histológico típico de neumonía organizativa; el cuadro remitió de forma completa tres meses después de la suspensión del tratamiento con trastuzumab.

Parece que el riesgo de reacción pulmonar asociada a trastuzumab estaría incrementado en pacientes con complicaciones pulmonares previas debidas a su enfermedad o comorbilidad asociada. Estas reacciones pueden manifestarse en forma de infiltrado pulmonar, síndrome de distrés respiratorio agudo, neumonitis, derrame pleural, edema pulmonar agudo e insuficiencia respiratoria. Es importante descartar el fallo cardíaco dado que la cardiotoxicidad es uno de los efectos secundarios que limita el uso de trastuzu$\mathrm{mab}^{9}$. Se ha observado insuficiencia cardíaca (NYHA clase II-IV) en pacientes tratados con trastuzumab en monoterapia o en combinación con taxanos, especialmente tras quimioterapia con una antraciclina. En casi todos los estudios de combinación se presentan casos de toxicidad cardíaca en forma de descenso de la FEVI: 23\% en el estudio de Montemurro $^{5}$ (2\% grado 3), $29 \%$ en el estudio de Esteva ${ }^{2}$ (3\% grado 3). En el ensayo M77001 hubo más descensos de la FEVI asociados al tratamiento de combinación que a docetaxel solo (17\% frente al $8 \%$ ). Por lo tanto durante el tratamiento con esta combinación es necesaria la monitorización de la FEVI y descartar esta complicación como origen de un infiltrado pulmonar.

El diagnóstico definitivo de un infiltrado pulmonar durante el tratamiento con trastuzumab puede requerir la realización de una biopsia pulmonar pero debe valorarse en cada caso ya que dependerá del estado clínico del paciente y de las ventajas que pueda implicar desde el punto de vista pronóstico y terapéutico.

El objetivo fundamental del tratamiento consiste en evitar la exposición a trastuzumab y suprimir el componente inflamatorio. La suspensión del fármaco puede ser suficiente; no obstante en algunos casos puede ser necesaria la instauración de tratamiento con glucocorticoides. Se desconoce si está indicado o no continuar con trastuzumab una vez la paciente se ha recuperado de su toxicidad pulmonar aguda.

En conclusión, la aparición de un infiltrado pulmonar difuso en un paciente en tratamiento con trastuzumab y en el que se han descartado otras causas más frecuentes de este cuadro, debe plantear la sospecha diagnóstica de toxicidad farmacológica y obliga a la supensión inmediata de tratuzumab y, si la situación clínica lo requiere, al inicio de tratamiento con glucocorticoides.

\author{
Correspondencia: \\ Dra. L. Heras López. \\ Servicio de Oncología \\ Hospital General de l' Hospitalet \\ Av. Josep Molins, 29-41 \\ E-08906 L'Hospitalet de Llobregat (Barcelona) \\ E-mail: 1heras02@yahoo.es
}




\section{Bibliografía}

1. Burris HA. Docetaxel plus trastuzumab in breast cancer. Seminars in Oncology 2001;1: 38-44.

2. Esteva FJ, Valero V, Booser D, et al. Phase II study of weekly docetaxel and trastuzumab for patients with HER-2overexpressing metastasic breast cancer. Journal of Clinical Oncology 2002; 7: 1800-1808.

3. Raff JP, Laskmini R, Umekalsoom M, et al. Phase II study of weekly docetaxel alone or in combination with trastuzumab in patients with metastasic breast cancer. Clinical Breast Cancer 2004; 4 (6): 420-47.

4. Tedesco KL, Thor AD, Johnson DH, et al. Docetaxel combined with trastuzumab is an active regimen in HER-2 $3+$ overexpressing and fluorescent in situ hybridization-positive metastatic breast cancer: a multi-institutional phase II trial. Journal of Clinical Oncology 2004; 6: 1071-1077.

5. Montemurro F, Choa G, Faggiuolo R, et al. A phase II study of three-weekly docetaxel and weekly tratuzumab in HER2Overexpressing advanced breast cancer.Oncology 2004; 66: 38-45.

6. Extra JM, Cognetti F, Maraninchi O, et al. Trastuzumab plus docetaxel versus docetaxel alone as first line treatment of HER2-positive metastatic breast cancer: results of a randomised multicentre trial. Eur J Cancer 2004; 2 (abstract 239).

7. Burstein HJ, Manola J, Younger J, et al. Docetaxel administered on a weekly basis for metastasic breast cancer. Journal of Clinical Oncology 2000; 6: 1212-1219.

8. Radzikowska E, Szczepulska E, Chabowski I, et al. Organising pneumonia caused by trastuzumab therapy for breast cancer. European Respiratory Journal 2003; 21: 552-555.

9. Perez E, Rodeheffer R. Clinical cardiac tolerability of trastuzumab. J Clin Oncol 2004; 22 (2): 322-329. 